\title{
REMARQUES SUR LES MOYENNES DES FONCTIONS DE PILTZ SUR LES ENTIERS FRIABLES
}

\author{
SARY DRAPPEAU
}

\begin{abstract}
We study the mean value of generalized divisor functions $\tau_{\kappa}(n)$ over integers without large prime factors (here $\kappa>0$ ). We relate this problem to the computation of the ratio $\Psi\left(x^{1 / \kappa}, y\right)^{\kappa} / \Psi(x, y)$, involving the $y$-smooth numbers counting function. We establish an inverse theorem, giving limitations on the range in $(x, y)$ in which this ratio can be asymptotically estimated uniformly in $\kappa$.
\end{abstract}

\section{INTRODUCTION}

Un entier $n$ est dit $y$-friable si son plus grand facteur premier $P(n)$ est inférieur ou égal à $y$, avec la convention $P(1)=1$. On note

$$
S(x, y):=\{n \leq x: P(n) \leq y\}, \quad \Psi(x, y):=|S(x, y)| .
$$

Nous nous intéressons ici aux valeurs moyennes

$$
\mathcal{M}_{f}(x, y):=\frac{1}{\Psi(x, y)} \sum_{n \in S(x, y)} f(n)
$$

d'une fonction multiplicative $f$ sur les entiers friables. Ces moyennes ont été étudiées dans la série de travaux [TW03, HTW08, TW08a, TW08b] ainsi que dans [BT05b], et jouent un rôle important dans la compréhension du cas $y=x$, par le biais de la factorisation de chaque entier $n=a b$ avec $P(a) \leq y$ et $p \mid b \Rightarrow p>y$ (voir par exemple [GS01]).

Notons $\tau(n)$ le nombre de diviseurs de $n$. Dans l'article [Dra15, faisant suite aux travaux de Fouvry-Tenenbaum [FT90, il est établi que

$$
\frac{1}{\Psi(x, y)} \sum_{\substack{n \in S(x, y) \\ n>1}} \tau(n-1) \sim \log x \quad\left(2 \leq y \leq x, \frac{\log \log x}{\log y} \rightarrow \infty\right) .
$$

Bien sûr, la fonction $n \mapsto \tau(n-1)$ n'est pas multiplicative ; ce problème est donc de nature très différente, et a priori plus délicate, que l'étude de

$$
\mathcal{M}_{\tau}(x, y)=\frac{1}{\Psi(x, y)} \sum_{n \in S(x, y)} \tau(n)
$$

On pourrait donc naïvement s'étonner que le comportement asymptotique de $\mathcal{M}_{\tau}(x, y)$ n'est en revanche connu que dans un domaine du type

$$
\exp \left\{\left(\log _{2} x\right)^{5 / 3+\varepsilon}\right\} \leq y \leq x,
$$

voir par exemple $\underline{\operatorname{Smi93}}]^{1}$. Ce domaine est plus restreint que 1.2 ; l'objectif de la présente note est d'éclaircir les raisons de cette discrépance.

Étant donné $\kappa>0$, nous considérons la fonction de Piltz $\tau_{\kappa}$, définie par la série génératrice

$$
\sum_{n \geq 1} \frac{\tau_{\kappa}(n)}{n^{s}}=\zeta(s)^{\kappa} \quad(\mathfrak{R e} s>1) .
$$

Date: 1st July 2016.

2010 Mathematics Subject Classification. Primary 11N25; Secondary 11 N37.

${ }^{1}$ L'estimation générale du Théorème 2.5 de [BT05b], appliquée au cas $f=\tau$, ne fournit pas un équivalent asymptotique. 
C'est une fonction multiplicative, avec $\tau_{\kappa}(p)=\kappa$ pour $p$ premier. Pour $\kappa=2$, nous retrouvons $\tau_{2}=\tau$. Nous avons également besoin de la notation suivante : lorsque $2 \leq y \leq x$, l'on note $\alpha(x, y)$ l'unique solution réelle positive à l'équation

$$
\log x=\sum_{p \leq y} \frac{\log p}{p^{\alpha}-1}
$$

Enfin, on pose

$$
u:=\frac{\log x}{\log y}, \quad \phi_{2}(s, y):=\sum_{p \leq y} \frac{(\log p)^{2} p^{s}}{\left(p^{s}-1\right)^{2}} \quad(2 \leq y \leq x, \mathfrak{R} \mathfrak{e}(s)>0) .
$$

Notre première observation est que dans le cas $f=\tau_{k}$, la valeur moyenne (1.1) peut être comparée de façon simple à $\Psi\left(x^{1 / \kappa}, y\right)^{\kappa}$.

Theorème 1. Pour $\kappa>0$ et $2 \leq y \leq x$, on $a$

$$
\sum_{n \in S(x, y)} \tau_{\kappa}(n)=A_{\kappa}(x, y) \Psi\left(x^{1 / \kappa}, y\right)^{\kappa}\left\{1+O_{\kappa}\left(\frac{1}{u}+\frac{\log y}{y}\right)\right\}
$$

avec

$$
A_{\kappa}(x, y)=\kappa^{-\frac{1}{2}}\left(\beta \sqrt{2 \pi \phi_{2}(\beta, y)}\right)^{\kappa-1} \quad\left(\beta=\alpha\left(x^{1 / \kappa}, y\right)\right) .
$$

Lorsque $\min \{y, u\} \rightarrow \infty$, on a l'approximation explicite

$$
A_{\kappa}(x, y) \sim \kappa^{\kappa / 2-1}\left(2 \pi u\left(1+\frac{\log x}{\kappa y}\right)\right)^{(1-\kappa) / 2}\left(\log \left(1+\frac{\kappa y}{\log x}\right)\right)^{1-\kappa} .
$$

Dans le cas $\kappa=2$, le problème s'interprète comme le comptage des points $(n, m) \in \mathbf{N}^{2}$, à coordonnées $y$-friables, situés sous l'hyperbole $m n=x$. Le résultat précédent met en valeur la pertinence de comparer le nombre total au nombre de points dans le carré $\max \{m, n\} \leq x^{\frac{1}{2}}$, plutôt qu'au nombre de points sur la droite $n=1$.

L'estimation (1.5), dont la preuve est très simple, permet d'ouvrir la voie à des estimations semi-asymptotiques [BT05b, Théorème 2.4], c'est-à-dire des estimations uniformes du rapport $\mathcal{M}_{\tau_{k}}(x / d, y) / \mathcal{M}_{\tau_{k}}(x, y)$ pour $1 \leq d \leq x$. Nous nous contentons d'illustrer cela par l'exemple

$$
\mathcal{M}_{\tau_{k}}(x / 2, y) \sim 2^{-\beta} \mathcal{M}_{\tau_{k}}(x, y) \quad\left(\beta=\alpha\left(x^{1 / \kappa}, y\right), x \rightarrow \infty\right),
$$

qui découle immédiatement des formules (1.5), 1.6] et du Théorème III.5.22 de [Ten15]. Il est utile de noter que

$$
\beta=o(1) \Leftrightarrow y \leq(\log x)^{1+o(1)}, \quad \beta=1+o(1) \Leftrightarrow \log y / \log _{2} x \rightarrow \infty .
$$

Dans le cas d'une fonction multiplicative $f$ plus générale, il est connu [BT05a, p.542] que les valeurs $f\left(p^{2}\right)$ (resp. $f\left(p^{\nu}\right)$ ) influent de façon essentielle sur l'ordre de grandeur de la valeur moyenne 1.1) dès lors que $y \leq(\log x)^{2+o(1)}\left(\operatorname{resp} . y \leq(\log x)^{\nu /(\nu-1)+o(1)}\right)$. De plus, dans les cas les plus favorables, une méthode de convolution permet facilement de se ramener à $f=\tau_{\kappa}$. Ce sont les raisons pour lesquelles nous ne tentons pas ici d'énoncer un résultat pour une fonction multiplicative générique $f$.

Notre second résultat concerne l'évaluation du rapport

$$
\frac{\Psi\left(x^{1 / \kappa}, y\right)^{\kappa}}{\Psi(x, y)} .
$$

Nous posons

$$
\phi_{0}(s, y):=\log \zeta(s, y)=-\sum_{p \leq y} \log \left(1-p^{-s}\right) \quad(\mathfrak{R e}(s)>0) .
$$

Notons que $\phi_{0}(\alpha(x, y), y) \sim u+\log \log y \operatorname{lorsque} \min \left\{\frac{y}{\log x}, u\right\} \rightarrow \infty$. 
Theorème 2. Soit $\kappa>0$. Alors on a

$$
\frac{\Psi\left(x^{1 / \kappa}, y\right)^{\kappa}}{\Psi(x, y)}=B_{\kappa}(x, y) \exp \left\{\int_{1}^{\kappa} \phi_{0}\left(\alpha\left(x^{1 / \lambda}, y\right), y\right) \mathrm{d} \lambda\right\}\left\{1+O_{\kappa}\left(\frac{1}{u}+\frac{\log y}{y}\right)\right\}
$$

avec

$$
B_{\kappa}(x, y)=\frac{\alpha \sqrt{2 \pi \phi_{2}(\alpha, y)}}{\left(\beta \sqrt{2 \pi \phi_{2}(\beta, y)}\right)^{\kappa}} \quad\left(\beta=\alpha\left(x^{1 / \kappa}, y\right)\right) .
$$

En particulier, on a

$$
B_{\kappa}(x, y) \sim \begin{cases}(2 \pi u)^{(1-\kappa) / 2} \kappa^{\kappa / 2}, & (y / \log x \rightarrow \infty), \\ (2 \pi y / \log y)^{(1-\kappa) / 2}, & (y / \log x \rightarrow 0) .\end{cases}
$$

Lorsque $(\log x)^{1+\varepsilon} \leq y \leq x$, une forme forte de la formule de Mertens (cf. [HT86, p.289]) permet d'écrire

$$
\begin{aligned}
& \int_{1}^{\kappa} \phi_{0}\left(\alpha\left(x^{\frac{1}{\lambda}}, y\right), y\right) \mathrm{d} \lambda \\
& \quad=(\kappa-1) \log \left(\mathrm{e}^{\gamma} \log y\right)+\int_{1}^{\kappa} \Xi\left(\frac{u}{\lambda}\right) \mathrm{d} \lambda+O_{\kappa, \varepsilon}\left(\frac{\log (u+1)}{\log y}+\frac{u}{\mathrm{e}^{(\log y)^{3 / 5-\varepsilon}}}\right) .
\end{aligned}
$$

Ici, $\xi(t)$ dénote l'unique solution non nulle de $\mathrm{e}^{\xi}=1+t \xi$ lorsque $t \neq 1$, avec $\xi(1)=0$, tandis que

$$
\Xi(u):=\int_{1}^{u} t \xi^{\prime}(t) \mathrm{d} t .
$$

Le second terme d'erreur dans (1.9) provient de la région sans zéros de $\zeta$ due à Korobov et Vinogradov [IK04, Corollary 8.30]. Il n'est uniformément borné que dans un domaine essentiellement du type $\left(H_{\varepsilon}\right)$. Cela suggère une limitation à l'estimation du rapport (1.7) en dehors du domaine $\left(H_{\varepsilon}\right)$ et constitue la motivation à ce qui suit.

Notre troisième résultat concerne un théorème inverse. Hildebrand [Hil84] a démontré que la validité, pour tout $\varepsilon>0$ fixé, de l'estimation

$$
\Psi(x, y)=x \rho(u) \mathrm{e}^{O_{\varepsilon}\left(y^{\varepsilon}\right)} \quad\left(y \geq(\log x)^{2+\varepsilon}\right),
$$

est équivalente à l'hypothèse de Riemann. Ici, $\rho(u)$ est la fonction de Dickman ( $c f$. [Ten15, Section III.5.3]). Sa méthode repose de façon essentielle sur une certaine équation fonctionnelle vérifiée par $\Psi(x, y)$ (voir également [Hil86]).

Compte tenu du Théorème 2, il est naturel de s'attendre à ce qu'un phénomène analogue se produise dans le cas du rapport (1.7).

Conjecture 1. Soit $\kappa \neq 1$ fixé. Supposons que pour tout $\varepsilon>0$, l'on ait

$$
\frac{\Psi\left(x^{1 / \kappa}, y\right)^{\kappa}}{\Psi(x, y)}=\frac{\rho(u / \kappa)^{\kappa}}{\rho(u)} \exp \left\{O_{\varepsilon}\left(y^{\varepsilon}\right)\right\} \quad\left((\log x)^{2+\varepsilon} \leq y \leq x\right) .
$$

Alors l'hypothèse de Riemann est vraie.

La quantité au membre de gauche de $(1.10)$ ne semble pas être facilement abordable par la méthode de Hildebrand Hil84. Si l'on autorise $\kappa$ à varier sur un intervalle non vide sans que cela n'altère la constante implicite dans (1.10), nous obtenons par une autre méthode le résultat suivant.

Theorème 3. Soit $\mathcal{I} \subset \mathbf{R}_{+}^{\times}$un ouvert non vide. Si l'estimation 1.10 est vraie uniformément pour $\kappa \in \mathcal{I}$, alors $\zeta(s) \neq 0$ lorsque $\mathfrak{R e}(s)>\frac{3}{4}$.

Nous précisons que notre approche est différente de celle de [Hil84, et consiste à raisonner directement à partir de l'estimation du Théorème 2. En particulier, nous n'utilisons pas l'identité de Hildebrand [Hil86]. 
Nous concluons en remarquant que le Théorème 3 fournit une autre démonstration de la version affaiblie suivante de [Hil84, Theorem 1] :

$$
\begin{aligned}
& \left(\forall \varepsilon>0, \quad \Psi(x, y)=x \rho(u) \mathrm{e}^{O_{\varepsilon}\left(y^{\varepsilon}\right)} \quad \text { pour }(\log x)^{2+\varepsilon} \leq y\right) \\
& \Longrightarrow \quad \zeta(s) \neq 0 \quad\left(\mathfrak{R e}(s)>\frac{3}{4}\right) .
\end{aligned}
$$

Remerciements. L'auteur remercie $\mathrm{R}$. de la Bretèche pour des remarques sur une version préliminaire.

Notations et rappels. Nous rappelons quelques définitions de [HT86]. La série de Dirichlet associée aux entiers $y$-friables est notée

$$
\zeta(s, y):=\sum_{P(n) \leq y} n^{-s}=\prod_{p \leq y}\left(1-p^{-s}\right)^{-1} \quad(\Re \mathfrak{R}(s)>0) .
$$

Nous rappelons la définition 1.3 du point-selle $\alpha(x, y)$. Nous définissons également

$$
\phi_{1}(s, y)=\frac{\mathrm{d}}{\mathrm{d} s} \phi_{0}(s, y)=-\sum_{p \leq y} \frac{\log p}{p^{s}-1} \quad(\mathfrak{R e}(s)>0) .
$$

Nous rappelons que $\phi_{2}(s, y)$, la dérivée seconde de $\phi_{0}(s, y)$, est définie en (1.4). Avec l'abbréviation $\alpha=$ $\alpha(x, y)$, le résultat principal de [HT86] est l'estimation

$$
\Psi(x, y)=\frac{x^{\alpha} \zeta(\alpha, y)}{\alpha \sqrt{2 \pi \phi_{2}(\alpha, y)}}\left\{1+O\left(\frac{1}{u}+\frac{\log y}{y}\right)\right\} \quad(2 \leq y \leq x) .
$$

Nous rappelons enfin l'estimation [HT86, Lemma 4]

$$
(-1)^{j} \phi_{j}(\alpha, y) \asymp u(\log y)^{j}, \quad(\log x \ll y \leq x, j \in\{1,2\}) .
$$

\section{Démonstration des RÉSultats}

2.1. Démonstration du Théorème 1. Le Théorème 1 découle aisément des calculs de [HT86, une fois la remarque faite que

$$
x^{s} \zeta(s, y)^{\kappa}=\left(x^{s / \kappa} \zeta(s, y)\right)^{\kappa} \quad(x, y \geq 2, \mathfrak{R e}(s)>0) .
$$

Ainsi, le rôle du point-selle est joué par la quantité $\beta(x, y)=\alpha\left(x^{1 / \kappa}, y\right)$. Des calculs semblables à ceux de [HT86] fournissent alors

$$
\sum_{n \in S(x, y)} \tau_{\kappa}(n)=\left\{1+O_{\kappa}\left(\frac{1}{u}+\frac{\log y}{y}\right)\right\} \frac{x^{\beta} \zeta(\beta, y)^{\kappa}}{\beta \sqrt{2 \kappa \pi \phi_{2}(\beta, y)}} .
$$

La comparaison de cette estimation avec (1.11) fournit le résultat annoncé (1.5). L'approximation (1.6) est déduite en utilisant [HT86, Corollary 1].

2.2. Démonstration du Théorème 2. Notons temporairement $\beta(\kappa)=\beta_{x, y}(\kappa)=\alpha\left(x^{1 / \kappa}, y\right)$. C'est une fonction dérivable de $\kappa>0$. Nous avons de plus

$$
\kappa \phi_{0}(\beta(\kappa), y)+\beta(\kappa) \log x-\phi_{0}(\beta(1), y)-\beta(1) \log x=\int_{1}^{\kappa} \phi_{0}(\beta(\lambda), y) \mathrm{d} \lambda \quad(\kappa>0) .
$$

Cette identité est aisément vérifiable en dérivant par rapport à $\kappa$, puis en utilisant la définition du point-selle (1.3). Le résultat annoncé est alors une conséquence simple de (2.1) et de [HT86, Theorem 1]. L'estimation (1.8) est une conséquence de [HT86, Corollary 1]. 
2.3. Démonstration du Théorème 3. Dans cette section, il sera utile d'abréger

$$
\alpha_{y}(u):=\alpha\left(y^{u}, y\right) \text {. }
$$

C'est une fonction dérivable de $u$; on note que pour $y \gg \log x$,

$$
\alpha_{y}^{\prime}(u)=-\frac{\log y}{\phi_{2}\left(\alpha_{y}(u), y\right)} \ll \frac{1}{u \log y} .
$$

En utilisant le Théorème 2, l'hypothèse 1.10 est réécrite sous la forme

$$
\left.\int_{1}^{\kappa} \phi_{0}\left(\alpha_{y}(u / \lambda), y\right), y\right) \mathrm{d} \lambda=\int_{1}^{\kappa} \Xi(u / \lambda) \mathrm{d} \lambda+O\left(y^{\varepsilon}\right) \quad\left(1 \leq u \leq y^{\frac{1}{2}-\varepsilon}, \kappa \in \mathcal{I}\right) .
$$

Nous voyons ceci comme une majoration de la différence $\phi_{0}\left(\alpha_{y}(u / \lambda), y\right)-\Xi(u / \lambda)$ en moyenne sur $\lambda$. La première étape est d'en déduire une majoration valable pour $\lambda$ fixé, autrement dit, retirer la moyenne sur $\lambda$. Cela utilise l'hypothèse supplémentaire sur $\mathcal{I}$, et c'est la raison pour laquelle nous obtenons in fine la valeur $\frac{3}{4}$ (plutôt que $\frac{1}{2}$ ). Nous montrons dans un premier temps que le Théorème 3 découle de la proposition suivante.

Proposition 1. Soit $\delta \in\left[0, \frac{1}{2}[\right.$. Supposons que pour tout $\varepsilon>0$, l'on ait

$$
\phi_{0}\left(\alpha_{y}(u), y\right)=\Xi(u)+O\left(y^{\delta+\varepsilon}\right) \quad\left((\log x)^{2+\varepsilon} \leq y \leq x\right) .
$$

Alors on a $\zeta(s) \neq 0$ pour $\mathfrak{R e}(s)>\frac{1}{2}+\delta$.

Démonstration que la Proposition 1 implique le Théorème 3. Soit $\kappa \in \mathcal{I}$, et $\left(\eta, \eta_{0}\right) \in \mathbf{R}^{2}$ des paramètres vérifiant $0<|\eta|<\eta_{0}$. Lorsque $\eta_{0}$ est suffisamment petit, on peut appliquer l'hypothèse $(2.2)$ avec $x$ remplacé par $x^{1 /(1+\eta)}$ et $\kappa$ remplacé par $\kappa /(1+\eta)$, ce qui fournit par soustraction

$$
\int_{1}^{1+\eta} \phi_{0}\left(\alpha_{y}(u / \lambda), y\right) \mathrm{d} \lambda=\int_{1}^{1+\eta} \Xi(u / \lambda) \mathrm{d} \lambda+O\left(y^{\varepsilon}\right)
$$

Soit $F(\eta)$ l'intégrale du membre de gauche. C'est une fonction $\mathcal{C}^{2}$ de $\eta$, qui vérifie de plus

$$
F^{\prime}(0)=\phi_{0}\left(\alpha_{y}(u), y\right), \quad F^{\prime \prime}(\eta)=-\frac{u}{(1+\eta)^{2}} \alpha_{y}^{\prime}\left(\frac{u}{1+\eta}\right) \phi_{1}\left(\alpha_{y}\left(\frac{u}{1+\eta}\right), y\right) \ll u .
$$

Les propriétés analogues sont vraies pour l'intégrale du membre de droite. En prenant les taux d'accroissement entre $\eta=0$ et $\eta=\eta_{0} u^{-\frac{1}{2}}$, nous obtenons, pour chaque $\varepsilon>0$ fixé,

$$
\phi_{0}\left(\alpha_{y}(u), y\right)=\Xi(u)+O\left(u^{\frac{1}{2}} y^{\varepsilon}\right) \quad\left(1 \leq u \leq y^{\frac{1}{2}-\varepsilon}\right) .
$$

Nous sommes donc en mesure d'appliquer la Proposition 1 avec $\delta=\frac{1}{4}$, ce qui fournit la conclusion annoncée.

La seconde étape consiste à extraire de l'hypothèse implicite $(2.3)$ une estimation asymptotique de $\phi_{0}(\beta, y)$ pour chaque $\left.\beta \in\right] \frac{1}{2}, 1[$. Nous montrons dans un deuxième temps que la Proposition 1 est une conséquence du lemme suivant, qui fait intervenir la fonction

$$
L(w):=\operatorname{li}(w)-\frac{w}{\log w} \quad(w>1)
$$

$\operatorname{avec} \operatorname{li}(w):=\int_{2}^{w} \mathrm{~d} t /(\log t)$.

Lemme 1. Soit $(\vartheta, \delta) \in \mathbf{R}^{2}$ avec $0<\delta<\vartheta<1$, et $F:[2,+\infty[\rightarrow] 1,+\infty[$ une fonction satisfaisant les conditions suivantes :

(i) F est continue par morceaux, continue à droite, et $F(y) \asymp y^{\vartheta}$,

(ii) on a

$$
L(F(y))=\int_{2}^{y} \frac{F(z)}{\log F(z)} \frac{\mathrm{d} z}{z \log z}+O\left(y^{\delta}\right) \quad(y \geq 2) .
$$

Alors on a

$$
F(y)=y^{\vartheta}+O\left(y^{\delta}(\log y)^{2}\right) \quad(y \geq 2)
$$


Démonstration que le Lemme 1 implique la Proposition 1 . Soit $\beta \in] \frac{1}{2}, 1-\delta[$ fixé. On définit

$$
F(y):=\exp \left\{\xi\left(\frac{-\phi_{1}(\beta, y)}{\log y}\right)\right\} \quad(y \geq 2) .
$$

Cela signifie que par définition,

$$
\frac{F(y)-1}{\log F(y)}=-\frac{\phi_{1}(\beta, y)}{\log y}
$$

On remarque que l'on a

$$
F(y) \sim \frac{-\phi_{1}(\beta, y)}{\log y} \log \left(\frac{-\phi_{1}(\beta, y)}{\log y}\right) \asymp y^{1-\beta}
$$

lorsque $y \rightarrow \infty$. Cela valide l'hypothèse $(i)$ avec $\vartheta=1-\beta$. L'hypothèse $(i i)$, quant à elle, découle de la relation (2.3). En effet, on a d'une part

$$
\Xi(u)=\operatorname{li}\left(\mathrm{e}^{\xi(u)}\right)+O(\xi(u)) \quad(u \geq 2) .
$$

D'autre part, une intégration par parties fournit

$$
\phi_{0}(\beta, y)=O_{\beta}(1)-\frac{\phi_{1}(\beta, y)}{\log y}-\int_{2}^{y} \frac{\phi_{1}(\beta, t)}{\log t} \frac{\mathrm{d} t}{t \log t} \quad(y \geq 2) .
$$

Les hypothèses du Lemme 1 étant vérifiées, on obtient pour tout $\varepsilon>0$ fixé,

$$
F(y)=y^{1-\beta}+O\left(y^{\delta+\varepsilon}\right) .
$$

D'autre part, par définition de $F$, on a

$$
\frac{F(y)-1}{\log F(y)}=\frac{1}{\log y} \sum_{p \leq y} \frac{\log p}{p^{\beta}-1}=\frac{1}{\log y}\left(\sum_{p \leq y} \frac{\log p}{p^{\beta}}+O_{\beta}(1)\right) .
$$

Ainsi la relation (2.6) implique

$$
\sum_{p \leq y} \frac{\log p}{p^{\beta}}=\frac{y^{1-\beta}}{1-\beta}+O_{\beta, \varepsilon}\left(y^{\delta+\varepsilon}\right) \quad(y \geq 2) .
$$

Le choix $\beta=\frac{1}{2}+\varepsilon$ implique alors $\sum_{p \leq y} \log p=y+O\left(y^{\frac{1}{2}+\delta+\varepsilon}\right)$, ce qui est classiquement équivalent à l'assertion que $\zeta(s) \neq 0$ pour $\mathfrak{R e}(s)>\frac{1}{2}+\delta$.

Démonstration du Lemme 1. Soient $\left(y_{1}, y_{2}\right) \in \mathbf{R}$ avec $10 \leq y_{1} \leq y_{2} \leq 2 y_{1}$. On note que $y_{1} \asymp$ $y_{2}$. Pour $w \geq F\left(y_{1}\right)$, on pose

$$
A_{y_{1}}(w):=\int_{F\left(y_{1}\right)}^{w} \frac{\mathrm{d} t}{(\log t)^{2}}=L(w)-L\left(F\left(y_{1}\right)\right) .
$$

On calcule de deux façons la quantité

$$
\mathcal{B}\left(y_{1}, y_{2}\right):=\frac{A_{y_{1}}\left(F\left(y_{2}\right)\right) \log F\left(y_{2}\right)}{F\left(y_{2}\right)}+\int_{F\left(y_{1}\right)}^{F\left(y_{2}\right)} \frac{\log w-1}{w^{2}} A_{y_{1}}(w) \mathrm{d} w .
$$

D'une part, une intégration par parties fournit immédiatement

$$
\mathcal{B}\left(y_{1}, y_{2}\right)=\log \log F\left(y_{2}\right)-\log \log F\left(y_{1}\right) .
$$

D'autre part, notre hypothèse 2.4 implique, pour tout $y^{\prime} \in\left[y_{1}, y_{2}\right]$,

$$
A_{y_{1}}\left(F\left(y^{\prime}\right)\right)=\int_{y_{1}}^{y^{\prime}} \frac{F(z)}{\log F(z)} \frac{\mathrm{d} z}{z \log z}+O\left(y_{2}^{\delta}\right) .
$$

Pour $w \in\left[F\left(y_{1}\right), F\left(y_{2}\right)\right]$, on définit $y_{w}:=\inf \{y \geq 2: F(y) \geq w\}$. Notre hypothèse $(i)$ implique ainsi $F\left(y_{w}\right) \geq w$, et $F\left(y_{w}-1\right)<w$. On en déduit que $\left|F\left(y_{w}\right)-w\right| \ll\left(\log y_{2}\right)^{2} y_{2}^{\delta}$, ce qui implique

$$
A_{y_{1}}(w)=\int_{y_{1}}^{y_{w}} \frac{F(z)}{\log F(z)} \frac{\mathrm{d} z}{z \log z}+O\left(y_{2}^{\delta}\right)
$$


d'après (2.8). Nous reportons cette estimation dans l'intégrale du membre de droite de (2.7). La contribution du terme d'erreur est encore une fois $O\left(y_{2}^{\delta-\vartheta} \log y_{2}\right)$. Par ailleurs, notre hypothèse (2.8) implique, pour tout $z \in\left[y_{1}, y_{2}\right]$,

$$
\left\{\begin{array}{l}
F(z)>w \Longrightarrow z \geq y_{w}+O\left(y_{2}^{\delta+1-\vartheta}\left(\log y_{2}\right)^{2}\right), \\
F(z) \leq w \Longrightarrow z \leq y_{w}+O\left(y_{2}^{\delta+1-\vartheta}\left(\log y_{2}\right)^{2}\right) .
\end{array}\right.
$$

On a donc

$$
\int_{F\left(y_{1}\right)}^{F\left(y_{2}\right)}\left(\int_{y_{1}}^{y_{w}} \mathbf{1}_{F(z)>w} \mathrm{~d} z+\int_{y_{w}}^{y_{1}} \mathbf{1}_{F(z) \leq w} \mathrm{~d} z\right) \mathrm{d} w \ll y_{2}^{1+\delta}\left(\log y_{2}\right)^{2} .
$$

Cela permet d'écrire

$$
\begin{aligned}
\int_{F\left(y_{1}\right)}^{F\left(y_{2}\right)} & \frac{\log w-1}{w^{2}} \int_{y_{1}}^{y_{w}} \frac{F(z)}{\log F(z)} \frac{\mathrm{d} z}{z \log z} \mathrm{~d} w \\
& =\int_{y_{1}}^{y_{2}} \frac{F(z)}{\log F(z)} \int_{F(z)}^{F\left(y_{2}\right)} \frac{\log w-1}{w^{2}} \mathrm{~d} w \frac{\mathrm{d} z}{z \log z}+O\left(y_{2}^{\delta-\vartheta} \log y_{2}\right) .
\end{aligned}
$$

Enfin, on remarque que

$$
\begin{aligned}
\frac{\log F\left(y_{2}\right)}{F\left(y_{2}\right)} \int_{y_{1}}^{y_{2}} \frac{F(z)}{\log F(z)} \frac{\mathrm{d} z}{z \log z} & +\int_{y_{1}}^{y_{2}} \frac{F(z)}{\log F(z)} \int_{F(z)}^{F\left(y_{2}\right)} \frac{\log w-1}{w^{2}} \mathrm{~d} w \frac{\mathrm{d} z}{z \log z} \\
& =\log \log y_{2}-\log \log y_{1} .
\end{aligned}
$$

On a donc montré que

$$
\begin{aligned}
\mathcal{B}\left(y_{1}, y_{2}\right) & =\log \log F\left(y_{2}\right)-\log \log F\left(y_{1}\right) \\
& =\log \log y_{2}-\log \log y_{1}+O\left(y_{2}^{\delta-\vartheta} \log y_{2}\right) \quad\left(10 \leq y_{1} \leq y_{2} \leq 2 y_{1}\right) .
\end{aligned}
$$

En sommant cela convenablement et par l'hypothèse $(i)$, nous obtenons l'estimation

$$
\log \log F(y)=\log \log y+\log \vartheta+O\left(y^{\delta-\vartheta} \log y\right) \quad(y \geq 10)
$$

dont la relation annoncée 2.5 est une conséquence.

\section{REFERENCES}

[BT05a] R. de la Bretèche and G. Tenenbaum, Entiers friables: inégalité de Turán-Kubilius et applications, Invent. Math. 159 (2005), no. 3, 531-588.

[BT05b] _ Propriétés statistiques des entiers friables, Ramanujan J. 9 (2005), no. 1-2, 139-202.

[Dra15] S. Drappeau, Théorèmes de type Fouvry-Iwaniec pour les entiers friables, Compos. Math. 151 (2015), no. $5,828-862$.

[FT90] É. Fouvry and G. Tenenbaum, Diviseurs de Titchmarsh des entiers sans grand facteur premier, Analytic number theory (Tokyo, 1988), Lecture Notes in Math., vol. 1434, Springer, Berlin, 1990, pp. 86-102.

[GS01] A. Granville and K. Soundararajan, The spectrum of multiplicative functions, Ann. of Math. (2) 153 (2001), no. 2, 407-470.

[Hil84] A. Hildebrand, Integers free of large prime factors and the Riemann hypothesis, Mathematika 31 (1984), no. 2, 258-271 (1985).

[Hil86] $\quad$ On the number of positive integers $\leq x$ and free of prime factors $>y$, J. Number Theory 22 (1986), no. 3, 289-307.

[HT86] A. Hildebrand and G. Tenenbaum, On integers free of large prime factors, Trans. Amer. Math. Soc. 296 (1986), no. 1, 265-290.

[HTW08] G. Hanrot, G. Tenenbaum, and J. Wu, Moyennes de certaines fonctions multiplicatives sur les entiers friables. II, Proc. Lond. Math. Soc. (3) 96 (2008), no. 1, 107-135.

[IK04] H. Iwaniec and E. Kowalski, Analytic number theory, American Mathematical Society Colloquium Publications, vol. 53, American Mathematical Society, Providence, RI, 2004.

[Smi93] H. Smida, Valeur moyenne des fonctions de Piltz sur les entiers sans grand facteur premier, Acta Arith. 63 (1993), no. 1, 21-50.

[Ten15] G. Tenenbaum, Introduction à la théorie analytique et probabiliste des nombres, $4^{\mathrm{e}}$ ed., Échelles, Belin, 2015, (English translation: Graduate Studies in Mathematics 163, Amer. Math. Soc. 2015). 
[TW03] G. Tenenbaum and J. Wu, Moyennes de certaines fonctions multiplicatives sur les entiers friables, J. Reine Angew. Math. 564 (2003), 119-166.

[TW08a] , Moyennes de certaines fonctions multiplicatives sur les entiers friables. III, Compos. Math. 144 (2008), no. 2, 339-376.

[TW08b] _ Moyennes de certaines fonctions multiplicatives sur les entiers friables. IV, Anatomy of integers, CRM Proc. Lecture Notes, vol. 46, Amer. Math. Soc., Providence, RI, 2008, pp. 129-141.

Aix Marseille Université, CNRS, Centrale Marseille, I2M UMR 7373, 13453 Marseille, France E-mail address: sary-aurelien.drappeau@univ-amu.fr 